\title{
Meta-analysis of the diagnostic value of serum, plasma and urine neutrophil gelatinase-associated lipocalin for the detection of acute kidney injury in patients with sepsis
}

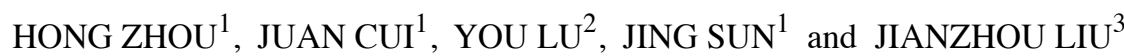 \\ Departments of ${ }^{1}$ Emergency and ${ }^{2}$ Pharmacy, Emergency General Hospital, Chaoyang, Beijing 100028; \\ ${ }^{3}$ Department of Cardiac Surgery, Peking Union Medical College Hospital, Beijing 100730, P.R. China
}

Received March 5, 2020; Accepted July 23, 2020

DOI: $10.3892 /$ etm.2021.9817

\begin{abstract}
The objective of the present study was to assess the diagnostic value of urine, serum and plasma neutrophil gelatinase-associated lipocalin (NGAL) for the early diagnosis of acute kidney injury (AKI) among patients with suspected sepsis. Therefore, a meta-analysis was carried out to evaluate diagnostic accuracy data from the literature regarding the diagnosis of AKI in patients with sepsis. Electronic databases were systematically searched for relevant studies and quality assessment was conducted using the Quality Assessment for Diagnostic Accuracy Studies 2 tool. A summary receiver operating characteristic curve analysis was performed, and several parameters including sensitivity, specificity, diagnosis odds ratio (DOR) and area under the curve (AUC) were calculated to evaluate the diagnostic performance of urine, serum and plasma NGAL. Meta-regression, sensitivity and subgroup analysis were also conducted to identify the source of heterogeneity in the eligible studies. In total, 28 studies were included. The pooled sensitivities for urine, serum and plasma NGAL were $0.87,0.83$ and 0.80 , respectively. Pooled specificity was $0.84,0.79$ and 0.74 . The DORs were 35,18 and 11 , respectively. The AUC for urine, serum and plasma NGAL were 0.92, 0.87 and 0.84 , respectively. Urine NGAL presented superior performance for the diagnosis of AKI with the highest AUC and other diagnostic accuracy values, compared with serum and plasma NGAL. Further studies are needed to clarify the controversial issue between the usefulness of serum and plasma NGAL.
\end{abstract}

Correspondence to: Dr Jianzhou Liu, Department of Cardiac Surgery, Peking Union Medical College Hospital, 1 Shuaifuyuan, Wangfujing, Dongcheng, Beijing 100730, P.R. China

E-mail: liujianzhou134@sina.com

Key words: sepsis, neutrophil gelatinase-associated lipocalin, acute kidney injury, meta-analysis

\section{Introduction}

Acute kidney injury (AKI; also referred to as acute kidney failure or acute renal failure) is defined as a sudden loss of renal function. AKI has a high incidence in critically-ill patients, particularly those in intensive care units (1). Delayed diagnosis of AKI may result in deterioration of renal function, long hospital stays or even death. In addition, sepsis has been consistently found to be an important contributing factor of AKI $(2,3)$. Mortality associated with septic AKI is significantly higher than that of non-septic AKI, and $>50 \%$ of AKI cases are considered septic $(4,5)$. Sepsis-induced AKI has been independently associated with an increased risk of longer hospital stays and mortality (6). Thus, due to the high morbidity and mortality of septic AKI, early prediction and diagnosis of this condition is essential.

Neutrophil gelatinase-associated lipocalin (NGAL) has been identified as a biomarker for AKI, as its concentration is significantly increased in urine, plasma and serum following ischemic kidney injury (7). NGAL is a $24-\mathrm{kDa}$ secreted glycoprotein, which was originally purified from a culture of murine kidney cells infected with simian virus $40(7,8)$. Zhang et al (6) demonstrated that NGAL was an independent predictor of AKI that is not influenced by age, sex, ethnicity, severity of injury and factors unrelated to renal function. Serum, urine and plasma are the main fluid types used in the diagnosis of AKI via the detection of NGAL (7). Previous studies have evaluated the diagnostic value of NGAL and its potential value in predicting outcomes of treatment, such as renal replacement therapy $(6,9)$ Although NGAL appears to hold promise in the prediction of AKI among patients with suspected sepsis, the diagnostic accuracy of NGAL according to sample source (serum, urine or plasma) is still unclear. In the present study, the diagnostic performance of NGAL for AKI was evaluated using a meta-analysis. The secondary objective of this study was to identify an optimal source of NGAL for the diagnosis of sepsis-induced AKI.

\section{Materials and methods}

Literature search. The present study was based on previously published articles and, as such, did not require ethics 
approval or patient consent. Eligible studies were identified using an electronic database search and by cross-checking the references of relevant papers up to June 2020. The PubMed, ScienceDirect, Web of Science, Embase, OVID, Cochrane Library and China National Knowledge Infrastructure databases were systematically searched using search terms, including 'neutrophil gelatinase-associated lipocalin', 'sepsis', 'severe sepsis and septic shock', 'acute kidney injury', 'serum NGAL', 'plasma NGAL' and 'urine NGAL', as well as their abbreviations and synonyms and all possible combinations. Moreover, references from the retrieved articles were also reviewed to identify additional relevant studies.

Study selection. Two investigators (JC and YL) independently extracted data from the eligible papers complying with the inclusion and exclusion criteria. Disagreements were subsequently reviewed and resolved through discussion. Studies were included in the analysis if they met the following inclusion criteria without time limitation:i) Use of serum, urine or plasma NGAL for prediction of AKI in patients with suspected sepsis; ii) stratification of septic patients into an AKI group and a non-AKI group; iii) definition of sepsis according to the standards of The American College of Chest Physicians/Society of Critical Care Medicine (ACCP/SCCM) (10), Society of CriticalCare Medicine/European Society of Intensive Care Medicine/American College of Chest Physicians/American Thoracic Society/Surgical Infection Society (SCCM/ESICM/ACCP/ATS/SIS) (11), or the Survival Sepsis Campaign 2012 consensus criteria (12); iv) definition of AKI according to the Risk, Injury, Failure, Loss, End-Stage Kidney Disease (RIFLE) (13), Acute Kidney Injury Network (AKIN) (14) or Kidney Disease Improving Global Outcomes (KDIGO) criteria (15); v) full-text articles written in English or Chinese; and vi) presence of detailed clinical data that can be used to calculate sensitivity and specificity, including and the number of true positive (TP), false positive (FP), true negative (TN) and false negative (FN) cases.

Exclusion criteria were defined as follows: i) Studies not including patients with sepsis; ii) studies not reporting data for the diagnostic accuracy of NGAL; and iii) studies providing insufficient data regarding the TP, FP, TN and FN numbers within the original published study. Only original articles were considered. Other publications, including letters, reviews, case reports or editorial articles were excluded. The two investigators reached a consensus on each item through discussion.

Data extraction and quality assessment. For each eligible study, the first author's name, year of publication, admission setting (intensive care unit or emergency room), country, study design, number of participants and the reference standards used to define sepsis and AKI were recorded. Diagnostic accuracy data for serum, urine and plasma NGAL, the area under the curve (AUC), optimal cut-off values, sensitivity, specificity and the number of true positive (TP), false positive (FP), true negative (TN) and false negative (FN) responses were recorded or calculated. The same two investigators (JC and YL) also evaluated the quality of the included studies in the data extraction process. The Quality Assessment for Diagnostic Accuracy studies-2 (QUADAS-2) tool by RevMan version 5.3 was used to assess the quality of eligible studies (16). The risk of bias for each item was graded as 'low', 'unclear' and 'high'. Two investigators appraised the study quality independently and discrepancies were resolved by discussion. Each study was ranked as having a high, low or unclear risk of bias according to four different areas: i) Patient selection; ii) index test; iii) reference standard; and iv) flow of patients through the study and timing of the index tests and reference standard (flow and timing).

Statistical analysis. TPs, TNs, FPs and FNs recorded in the included studies are the primary data used to assess the sensitivity, specificity, positive likelihood ratio (PLR), negative likelihood ratio (NLR), diagnostic odds ratios (DOR) and AUC using Stata (version 15.1). The diagnostic performance of serum, urine and plasma NGAL was determined by calculating pooled sensitivity, specificity, PLR, NLR and DOR, with 95\% CIs. Likelihood ratios (positive and negative likelihood ratio) are a ratios of the probabilities that a test result is correct to the probability that the test is incorrect [positive likelihood ratios=sensitivity/(1-specificity); negative likelihood ratios=specificity/(1-sensitivity)]. The larger the ratio of PLR, the greater probability of a true positive when the result is positive. The smaller the ratio of NLR, the greater probability of a true negative when the result is negative. The DOR $[(\mathrm{TP} \times \mathrm{TN}) /(\mathrm{FP} \times \mathrm{FN})]$ was used to reflect the relationship between diagnostic test and disease. Higher numbers would indicate improved performance in diagnosing patients with/without sepsis-AKI. Summary receiver operating characteristic (SROC) curves were generated to estimate the effect of sensitivity and specificity and were constructed based on TP and FP rates. TP and FP rates can be calculate through the following two formulas: TP rates=TP/(TP + FP) x100; FP rates $=\mathrm{FP} /(\mathrm{FP}+\mathrm{TN}) \times 100$. The area under the curve $(\mathrm{AUC})$ of the SROC was calculated to assess the performance of serum, urine and plasma NGAL individually.

The heterogeneity between studies was assessed using the $\chi^{2}$ test and the inconsistency index $\left(\mathrm{I}^{2}\right)$. An $\mathrm{I}^{2}>50 \%$ with $\mathrm{P}<0.05$ from the $\chi^{2}$ test is indicative of significant heterogeneity (17). In this case, a random effect model was chosen to pool the data of sensitivity, specificity and AUC. Otherwise, a fixed effect model was used.

The threshold effect is considered as a possible cause of heterogeneity in diagnostic accuracy analysis (18). Spearman correlation was used to analyse the logit of sensitivity and the logit of (1-specificity) and to verify the existence of threshold effect. A strong positive correlation (correlation $>0.6$ ) between sensitivity and (1-specificity), with $\mathrm{P}<0.05$ was considered to indicate a statistically significant threshold effect (18).

Sensitivity analyses and subgroup analyses were also conducted to determine if a certain variance could affect the heterogeneity and overall diagnostic effect. Sensitivity analysis was performed by omitting one study at a time to examine stability of the pooled results. Meta-regression and subgroup analyses were performed to identify factors that could influence heterogeneity and the overall diagnostic effect.

Publication bias was analyzed by using the Deeks' funnel plot and an asymmetry test (19). $\mathrm{P}<0.05$ is considered to indicate the existence of publication bias.

Statistical analysis was performed using Stata version 15.1 (StataCorp LP). Quality assessment of the included studies was conducted using RevMan version 5.3 (The Nordic Cochrane Centre; The Cochrane Collaboration). 


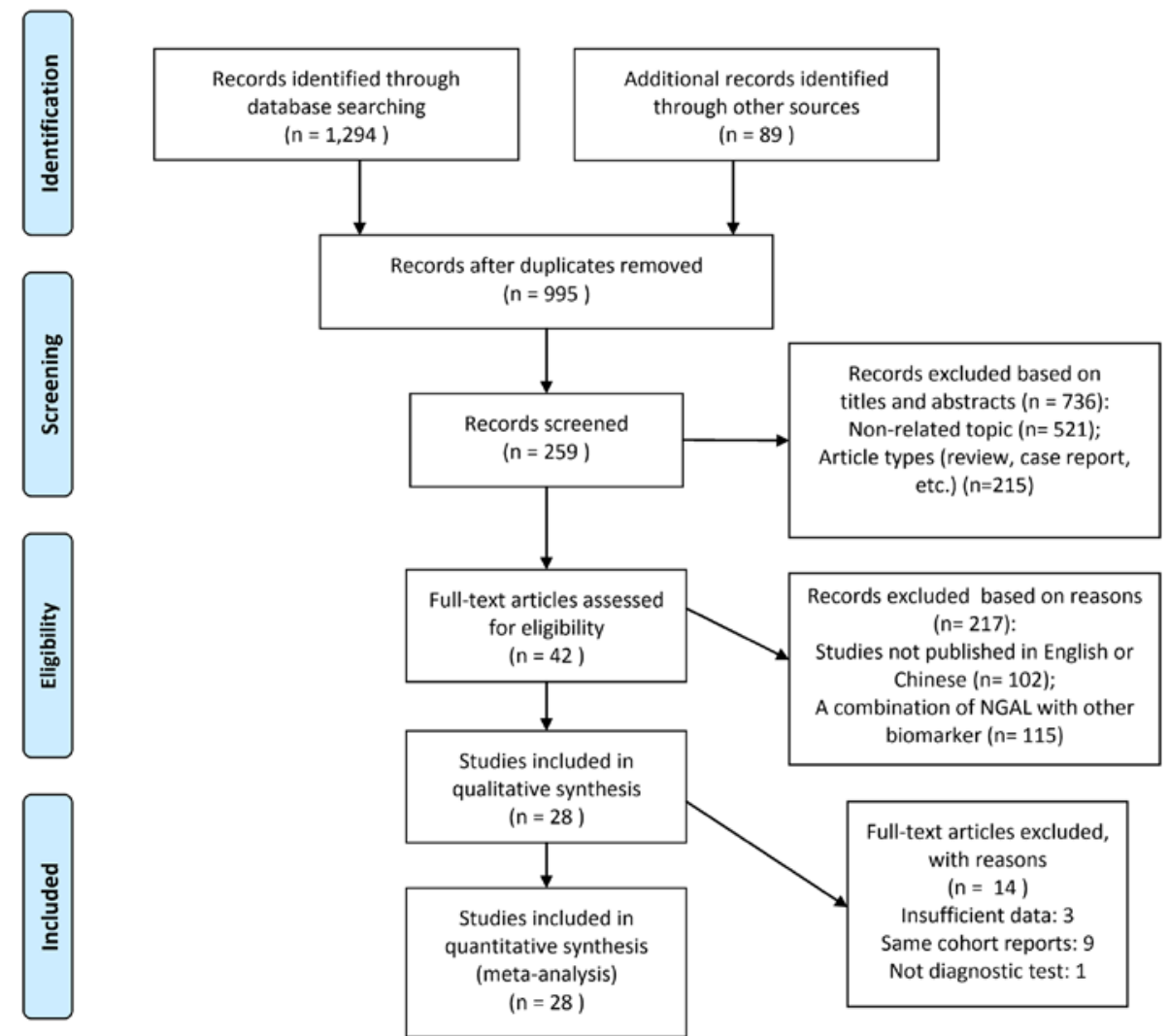

Figure 1. Flowchart of the literature search design and study selection. NGAL, neutrophil gelatinase-associated lipocalin.

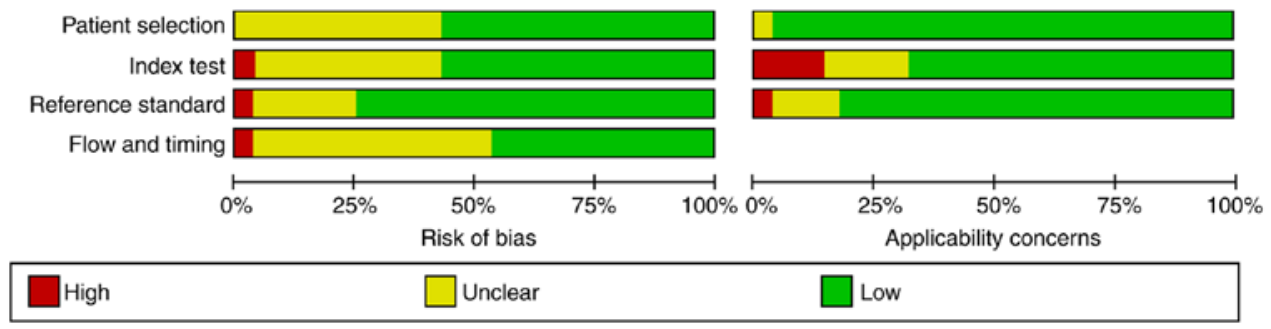

Figure 2. Risk of bias and applicability concerns graph of the 28 included studies.

\section{Results}

Study evaluation. Overall, 1,383 potential citations were identified for inclusion into the study through multiple database searches and cross-checking of reference lists. After removing duplicates, 338 studies were excluded. Of these, 259 articles were included based on titles and abstracts. A further 217 studies were excluded since they did not meet the eligibility of the present study and 42 studies remained as potential candidates for our meta-analysis. After reviewing the full text according to the inclusion and exclusion criteria, 28 studies were ultimately considered eligible and used for subsequent analysis $(5,13,20-45)$. The study selection process is illustrated in Fig. 1.

Study characteristics. The included studies were conducted on different continents (Europe, Asia, North and South America) over a period spanning 10 years (2010-2020). Of the 28 studies, 9 described the diagnostic performance of serum
NGAL $(5,20,21,24,28,31,34,37,41), 10$ of plasma NGAL (13, $23,25,27,32,35,38,39,43,44)$ and 14 of urine NGAL $(22,24$, 26,29-31,33,34,36,37,40,42,44,45). Collectively, these studies included a total of 2,561 participants. Prospective studies took up $~ 43 \%$ of the included studies. The samples of NGAL were taken in two admission settings (ICU or ER) and samples from ICU took up 24 studies of the included studies. The remaining studies were of ER. Detailed information regarding study design, sample sizes and reference standards from the included studies are included in Table I.

Quality assessment. The quality and potential bias of the studies were assessed using the QUADAS-2 tool. The 'risk of bias' tool evaluates four items, patient selection, index test, reference standard and flow and timing. High risk was mostly observed in the 'index test' category because many studies did not provide the interpretation method of the NGAL test results and did not provide a threshold. Detailed information of the included studies and the results of distribution are presented in Fig. 2. 


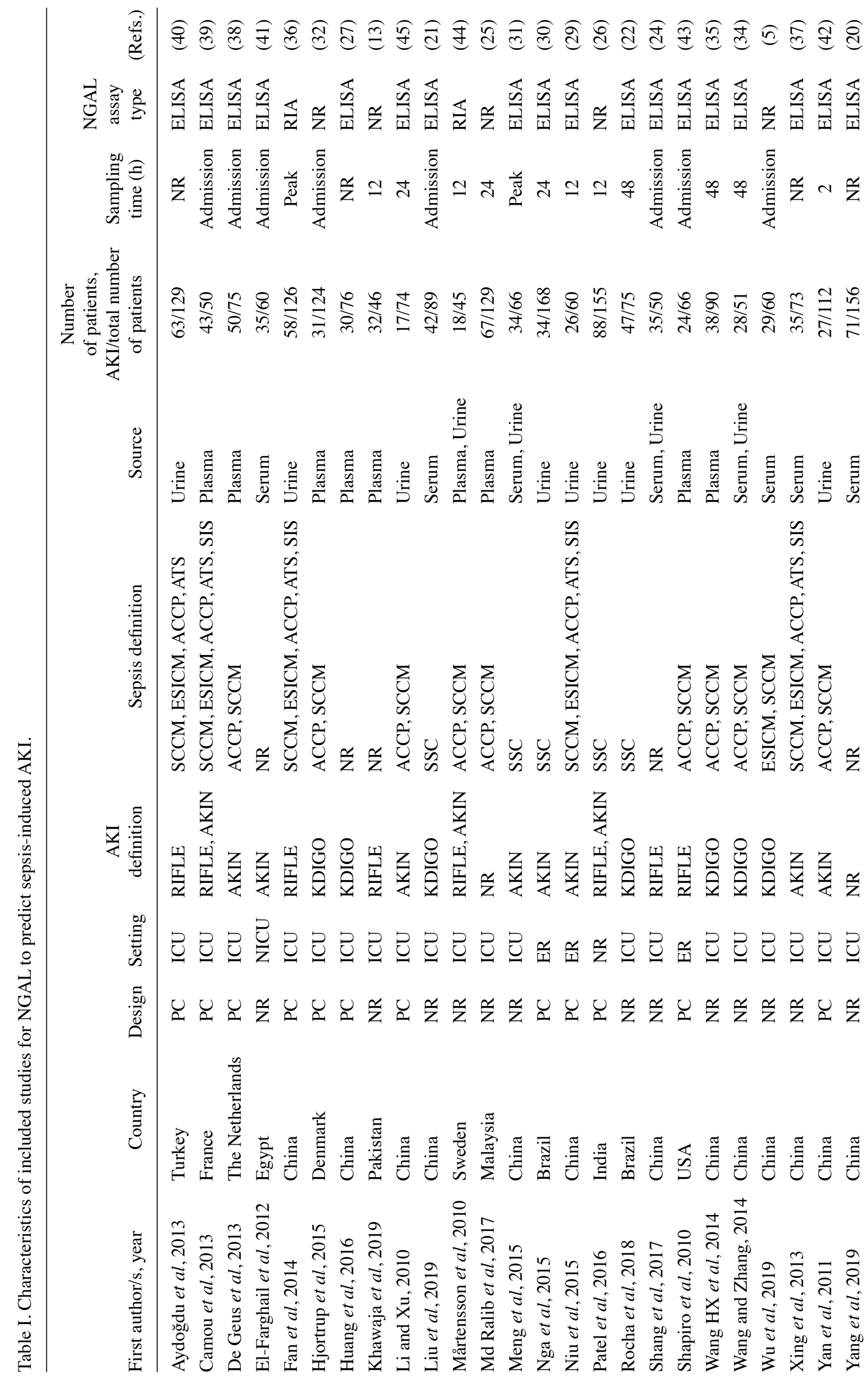



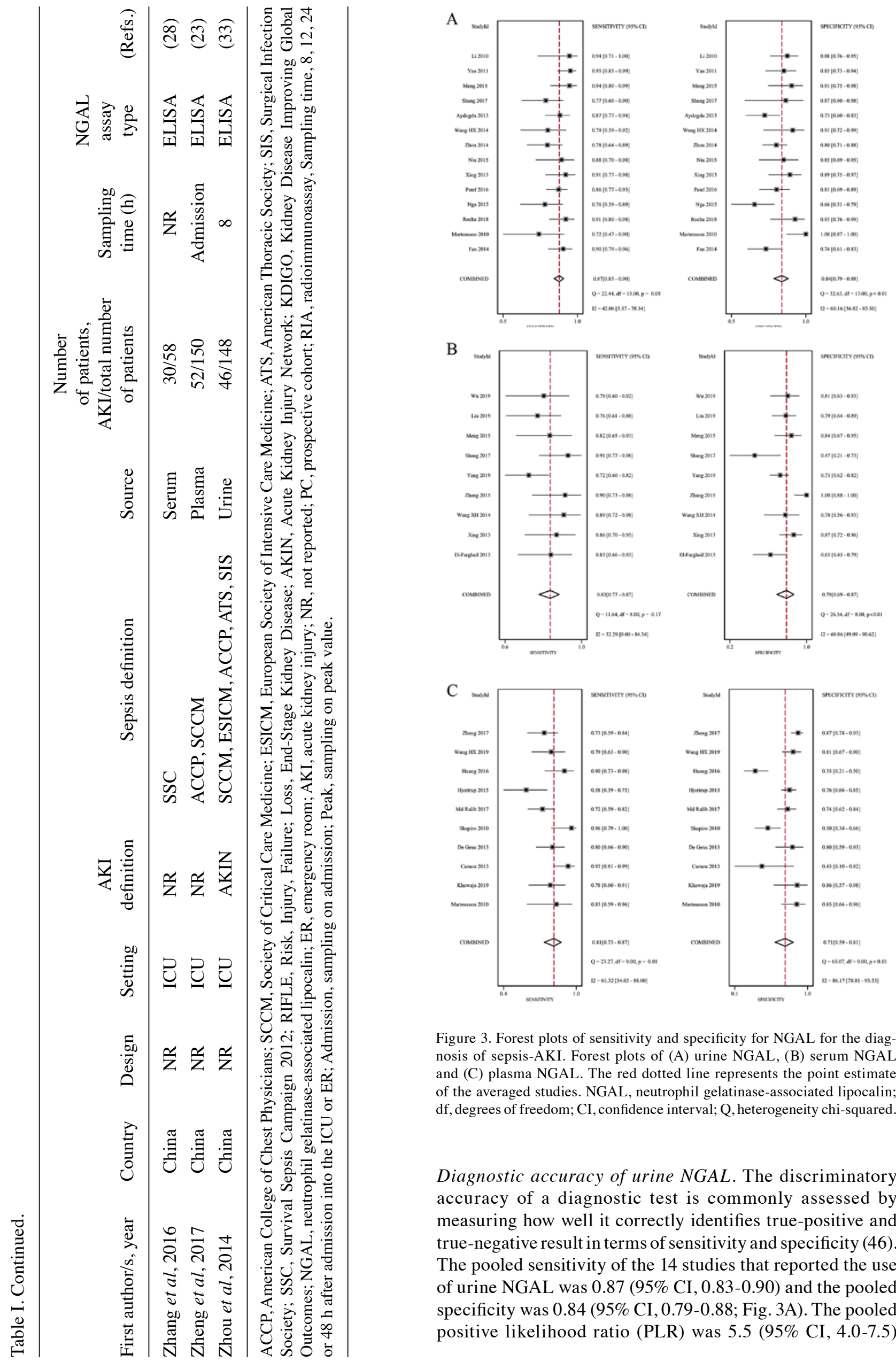

Figure 3. Forest plots of sensitivity and specificity for NGAL for the diagnosis of sepsis-AKI. Forest plots of (A) urine NGAL, (B) serum NGAL and (C) plasma NGAL. The red dotted line represents the point estimate of the averaged studies. NGAL, neutrophil gelatinase-associated lipocalin; df, degrees of freedom; CI, confidence interval; Q, heterogeneity chi-squared.

Diagnostic accuracy of urine NGAL. The discriminatory accuracy of a diagnostic test is commonly assessed by measuring how well it correctly identifies true-positive and true-negative result in terms of sensitivity and specificity (46). The pooled sensitivity of the 14 studies that reported the use of urine NGAL was 0.87 (95\% CI, 0.83-0.90) and the pooled specificity was 0.84 (95\% CI, 0.79-0.88; Fig. 3A). The pooled positive likelihood ratio (PLR) was 5.5 (95\% CI, 4.0-7.5) 


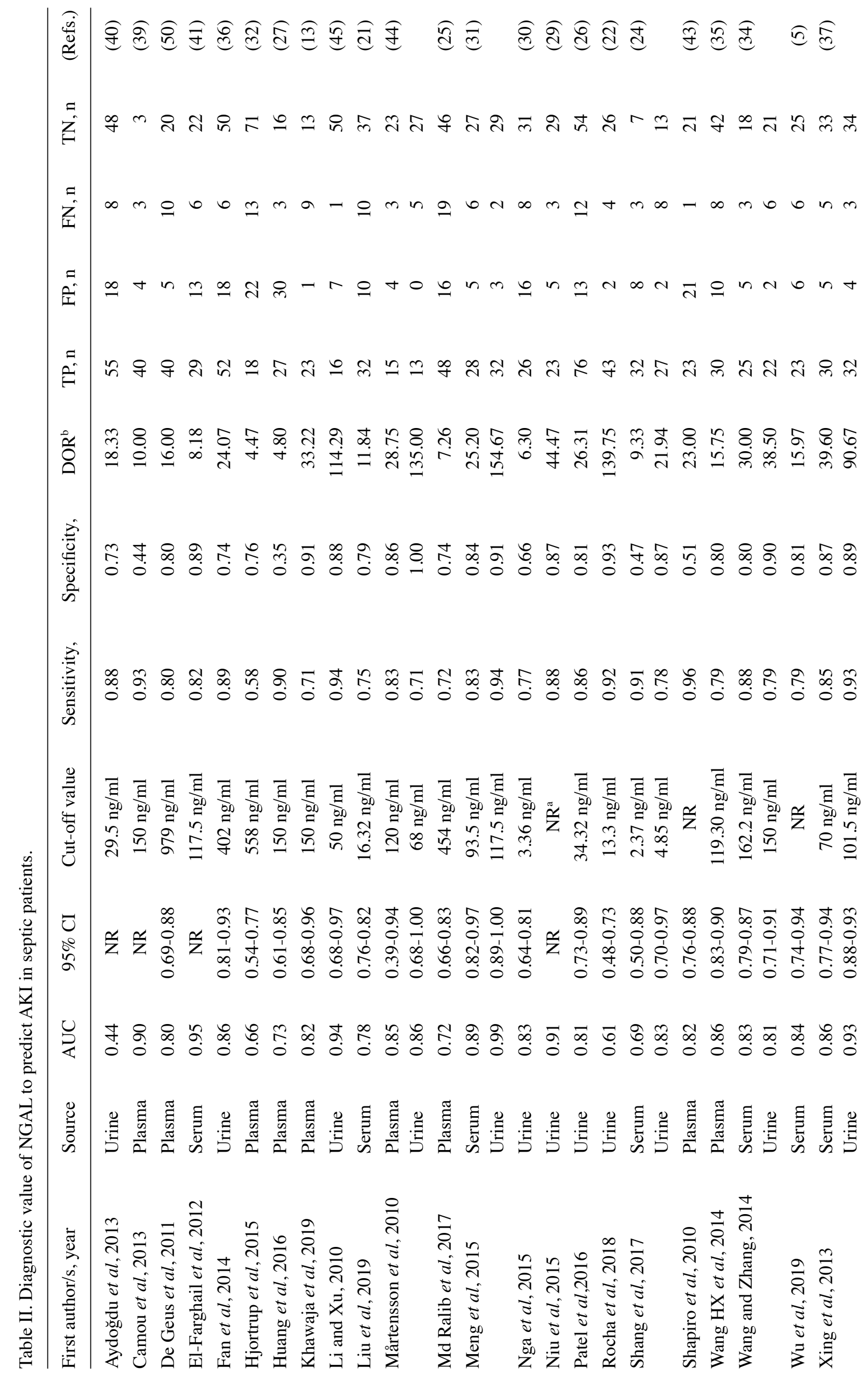




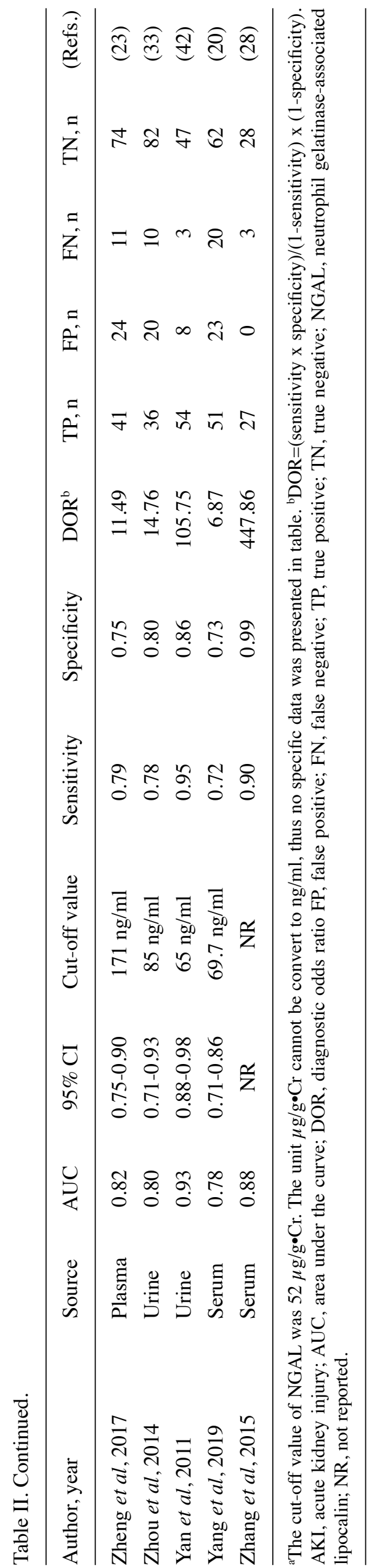
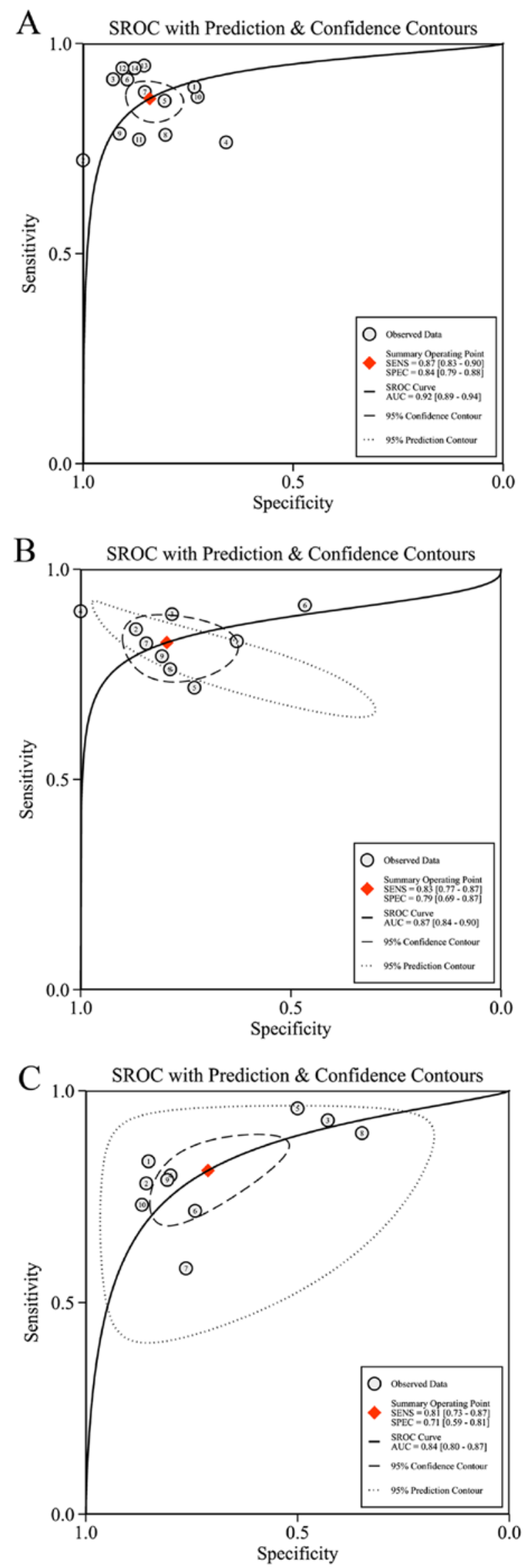

Figure 4. SROC for each type of NGAL assay. SROC curves of (A) urine NGAL, (B) serum NGAL and (C) plasma NGAL. SROC, summary receiver operating characteristic curve; NGAL, neutrophil gelatinase-associated lipocalin; AUC, area under the curve; SENS, sensitivity; SPEC, specificity. 
Table III. Result of meta-regression, subgroup analysis.

\begin{tabular}{lcccc}
\hline Source & Number of studies & Sensitivity $(95 \%$ CI $)$ & P-value & Specificity (95\% CI) \\
\hline Plasma NGAL & 3 & & & $0.71(0.52-0.91)$ \\
$\quad$ Prospective & 7 & $0.89(0.82-0.95)$ & $<0.01$ & $0.74(0.62-0.85)$ \\
Non-prospective & 7 & $0.76(0.70-0.82)$ & & $0.74(0.69-0.78)$ \\
Urine NGAL & 2 & $0.86(0.82-0.90)$ & $<0.01$ & $0.82(0.76-0.89)$ \\
Prospective & & $0.87(0.80-0.95)$ & & $<0.01$ \\
Non-prospective & 7 & & \\
\hline
\end{tabular}

Table IV. Result of sensitivity analysis.

A, Serum NGAL

\begin{tabular}{|c|c|c|c|c|c|c|}
\hline Included studies & Sensitivity & $\mathrm{I}^{2}(\%)$ & $\mathrm{P}$-value & Specificity & $\mathrm{I}^{2}(\%)$ & P-value \\
\hline All & $0.83(0.77-0.87)$ & 32.29 & 0.17 & $0.79(0.69-0.87)$ & 69.86 & 0.00 \\
\hline Without Zhang et al (23) & $0.82(0.76-0.86)$ & 22.40 & 0.25 & $0.75(0.68-0.82)$ & 53.70 & 0.03 \\
\hline
\end{tabular}

B, Urine NGAL

\begin{tabular}{|c|c|c|c|c|c|c|}
\hline Included studies & Sensitivity & $\mathrm{I}^{2}(\%)$ & P-value & Specificity & $\mathrm{I}^{2}(\%)$ & P-value \\
\hline All & $0.87(0.83-0.90)$ & 42.06 & 0.05 & $0.84(0.79-0.80)$ & 60.16 & 0.00 \\
\hline Without Mårtensson et al (44) & $0.88(0.84-0.91)$ & 38.37 & 0.08 & $0.82(0.78-0.86)$ & 52.28 & 0.01 \\
\hline
\end{tabular}

C, Plasma NGAL

\begin{tabular}{|c|c|c|c|c|c|c|}
\hline Included studies & Sensitivity & $\mathrm{I}^{2}(\%)$ & P-value & Specificity & $\mathrm{I}^{2}(\%)$ & P-value \\
\hline All & $0.81(0.73-0.87)$ & 61.32 & 0.01 & $0.71(0.59-0.81)$ & 86.17 & 0.00 \\
\hline Without Hjortrup et al (32) & $0.83(0.75-0.88)$ & 48.24 & 0.05 & $0.71(0.57-0.82)$ & 87.21 & 0.00 \\
\hline
\end{tabular}

and the pooled negative likelihood ratio (NLR) was 0.16 (95\% CI, 0.12-0.20; Table II). Using a random effect model, the DOR was 35 (95\% CI, 21-58). There was no threshold effect, as indicated by Spearman correlation analysis $(\rho=0.50 ; P=0.25$; data not shown). An SROC analysis was conducted to evaluate the diagnostic accuracy of urine NGAL (Fig. 4A). An AUC of 0.92 was obtained from the SROC curve, suggesting that urine NGAL achieved high diagnostic accuracy in diagnosing sepsis-AKI, due to the observation that AUC $>0.9$.

Estimation of the inconsistency index for sensitivity and specificity $\left(\mathrm{I}^{2}=42.06 \%\right.$ and $60.16 \%$, respectively; $\mathrm{P}=0.07$ and 0.01 , respectively; Fig. $3 \mathrm{~A}$ ) indicated that there was heterogeneity in the pooled specificity of urine NGAL between the included studies. Therefore, a subgroup analysis and meta-regression were conducted based on the study design (prospective and non-prospective; Table III). The study design significantly affected the results of sensitivity and specificity. Indeed, the use of a prospective design resulted in a significantly lower sensitivity $(\mathrm{P} \leq 0.01)$ and specificity $(\mathrm{P} \leq 0.01)$, compared with a non-prospective design.
Diagnosis accuracy of serum NGAL. A total of nine studies reported the use of serum NGAL. The pooled sensitivity for these studies in the diagnosis of sepsis-related AKI was 0.83 (95\% CI, 0.77-0.87), whereas the pooled specificity was 0.79 (95\% CI, 0.69-0.87; Fig. 3B). The pooled PLR was 4.00 (95\% CI, 2.6-6.2), while the pooled NLR was 0.22 (95\% CI, 0.16-0.30). The DOR was 18 (95\% CI, 9-36) with higher values indicating better discriminatory performance. An SROC analysis was carried out to evaluate the diagnostic accuracy of serum NGAL, demonstrating an AUC of 0.87 (95\% CI, 0.84-0.90; Fig. 4B). There was no notable threshold effect in the nine studies included in this meta-analysis $(\rho=0.34$; $\mathrm{P}=0.12$; data not shown).

An $\mathrm{I}^{2}$ of $32.29 \%$ for sensitivity $(\mathrm{P}=0.17)$ and $69.86 \%$ for specificity $(\mathrm{P}<0.001$; Fig. $3 \mathrm{~B})$ indicated that there was a significant heterogeneity in the pooled specificity in the included studies. A sensitivity analysis was conducted to eliminate factors that influence of heterogeneity. In the interpretation of the heterogeneity of the diagnostic accuracy of serum NGAL, sensitivity analysis suggested that the study by Zhang et al (28) had an impact on heterogeneity. Omitting the 
study by Zhang et al (28) decreased the $\mathrm{I}^{2}$ both in sensitivity (from 31.29 to $22.4 \% ; \mathrm{P}=0.25$ ) and specificity (from 69.86 to $53.70 \%$; $\mathrm{P}=0.03$; Table IV). The value of $\mathrm{I}^{2}$ in the pooled specificity reduced by $16 \%$ but the heterogeneity did not disappear.

Diagnostic accuracy of plasma NGAL. In total, 10 studies reported the use of plasma NGAL for AKI diagnosis. These studies had a pooled sensitivity of 0.81 (95\% CI, 0.73-0.87) and a pooled specificity of 0.71 (95\% CI, 0.59-0.81; Fig. 3C). The pooled PLR was 2.8 (95\% CI, 2.0-3.9) and the pooled NLR was 0.26 (95\% CI, 0.20-0.36). The DOR was 11 (95\% CI, 7-16), which was the worst performer among the three NGALs tested using a random effect model. In addition, the AUC obtained from SROC analysis was 0.84 (95\% CI, 0.80-0.87; Fig. 4C). There was no notable threshold effect in the meta-analysis ( $\mathrm{Q}=-0.93 ; \mathrm{P}=0.87$; data not shown). The estimation of sensitivity and specificity $\left(\mathrm{I}^{2}=61.32\right.$ and $86.17 \%$, respectively; $\mathrm{P}=0.01$ and $<0.001$, respectively; Fig. $3 \mathrm{C}$ ) indicated that there was heterogeneity between the studies. Thus, a subgroup analysis was performed based on study design (prospective and non-prospective). Meta-regression and subgroup analysis revealed that study design had a significant effect on the diagnostic accuracy of plasma NGAL. The use of a prospective design resulted in a significantly higher sensitivity $(\mathrm{P} \leq 0.01)$ but no effect on specificity $(\mathrm{P}=0.36)$, compared with those from a non-prospective design (Table III).

Evaluation of publication bias. Publication bias was analysed using a Deeks' funnel plot and an asymmetry test. No publication bias was detected among the studies in the urine $(\mathrm{P}=0.16)$, serum $(\mathrm{P}=0.052)$ and plasma NGAL $(\mathrm{P}=0.16)$ groups.

\section{Discussion}

The morbidity and mortality of patients with sepsis in intensive care units remain high (2). In addition, AKI is among the most severe complications of sepsis (4). NGAL is the most extensively researched biomarker for the diagnosis of AKI in blood and urine specimens. Zhang et al (6) previously examined the diagnostic values of plasma and urine NGAL using meta-analysis. However, the number of studies included in their analysis was limited, and the reported high diagnostic value for urine NGAL was later questioned by Törnblom et al (47). Moreover, in the Zhang et al (6) study, the role of serum NGAL in diagnosing AKI was not examined. Thus, the optimal source of NGAL for the diagnosis of sepsis-induced AKI remains unknown.

The aim of the present meta-analysis was to assess the diagnostic accuracy of serum, plasma and urine NGAL among patients with suspected sepsis. In the present study, urine NGAL presented improved performance compared with serum and plasma, with a relatively high sensitivity, specificity and DOR, as well as the highest AUC. The present findings further support the notion that urine NGAL can predict sepsis-related AKI, which is consistent with the prospective cohort studies carried out by da Rocha et al and Patel et al $(22,26)$. Urine NGAL is an independent predictor of AKI, as it is not affected by the presence of sepsis (48) By contrast, NGAL levels in the bloodstream of patients with sepsis may increase due to injury to the kidney, thereby influencing the diagnosis of AKI through plasma and serum specimens (49). Additionally, urine testing represents a convenient, fast and non-invasive collection technique. Thus, the majority of patients would be eligible for diagnosis of AKI through the urine, especially paediatric patients. Notably, compared with serum creatinine as a diagnostic reference, the time to diagnose AKI with urine NGAL is $2 \mathrm{~h}$ shorter (45).

In the present study, serum NGAL also demonstrated a relatively high AUC. Although only a limited number of studies included in the present meta-analysis reported the use of serum NGAL, a recent prospective cohort study has demonstrated that the combined performance of serum and urine NGAL can increase the early diagnostic accuracy of sepsis-induced AKI (50). Furthermore, previous studies by Meng et al and Xing et al $(31,37)$ demonstrated that the predictive performance of serum and urine NGAL for sepsis-related AKI increased significantly in combination compared with that in either alone. Notably, AKI diagnosis through serum NGAL would be available to patients suffering from oliguria or other conditions that cause inconvenience for urine sampling.

The use plasma NGAL for the prediction of AKI in septic patients remains controversial. There is no consensus on the predictive ability of plasma NGAL according to previous studies $(40,44)$. Mårtensson et al $(44)$ reported a poor predictive ability for plasma NGAL and suggested that NGAL levels in the plasma may be affected by other non-renal factors such as inflammation. Aydoğdu et al (40) demonstrated that concurrent sepsis could increase the levels of plasma NGAL in the absence of AKI and thus, the diagnostic accuracy of plasma NGAL may have been corrupted. By contrast, previous studies by Huang et al and Shapiro et al $(27,43)$ suggested that plasma NGAL could predict sepsis-related AKI. The different locations in which these studies were conducted may have led to the discrepancies in study outcomes, as suggested by $\mathrm{Md}$ Ralib et al (25). In the present meta-analysis, plasma NGAL was less useful than other NGAL sources in diagnosing sepsis-related AKI. Indeed, compared with urine and serum specimens, plasma NGAL presented the poorest sensitivity, specificity and AUC.

Nevertheless, the present study has certain limitations that should be taken into consideration when interpreting its findings. High heterogeneity performed in the meta-analysis for plasma NGAL was a substantial problem. Although sensitivity analysis omitted studies that affected heterogeneity, the results only changed slightly. Subgroup analyses were performed to identify factors that influenced heterogeneity, yet the causes of heterogeneity could not be determined. Moreover, as aforementioned, there is still a controversial in the use of plasma NGAL. Further prospective studies focusing on plasma NGAL should also be considered in the future.

In conclusion, the present findings suggested that urine NGAL was a robust diagnostic biomarker of AKI, with the highest sensitivity, specificity and AUC. However, each specimen type had its own advantages and weaknesses. For instance, the use of serum and plasma NGAL in combination may enhance the strengths and reduce the deficiencies of each, resulting in a more accurate diagnosis. Further studies are encouraged to provide more robust evidence. 


\section{Acknowledgements}

Not applicable.

\section{Funding}

No funding was received.

\section{Availability of data and materials}

All data generated or analyzed during this study are included in this published article.

\section{Authors' contributions}

$\mathrm{HZ}$ analysed the research data and was the major contributor in the preparation and writing of the manuscript. JC collected the original data and quality assessment and conducted the data processing of the meta-analysis. YL collected the original data and quality assessment. JS is responsible for interpretation of data for the work, visualization and tables and figures production. JL made substantial contributions to the conception and design of the study and drafted the manuscript. All authors read and approved the final manuscript.

\section{Ethics approval and consent to participate}

Not applicable.

\section{Patient consent for publication}

Not applicable.

\section{Competing interests}

The authors declare that they have no competing interests.

\section{References}

1. Lameire NH, Bagga A, Cruz D, De Maeseneer J, Endre Z, Kellum JA, Liu KD, Mehta RL, Pannu N, Van Biesen W and Vanholder R: Acute kidney injury: An increasing global concern. Lancet 382: 170-179, 2013.

2. Kellum JA, Wen X, de Caestecker MP and Hukriede NA: Sepsis-associated acute kidney injury: A problem deserving of new solutions. Nephron 143: 174-178, 2019.

3. Zarbock A, Gomez H and Kellum JA: Sepsis-induced acute kidney injury revisited: Pathophysiology, prevention and future therapies. Curr Opin Crit Care 20: 588-595, 2014.

4. Bellomo R, Kellum JA, Ronco C, Wald R, Martensson J, Maiden M, Bagshaw SM, Glassford NJ, Lankadeva Y, Vaara ST and Schneider A: Acute kidney injury in sepsis. Intensive Care Med 43: 816-828, 2017.

5. JX W, HS Z and XL C: Clinical significance of renal biomarkers for early evaluation of acute kidney injury in sepsis. Chin J Crit Care Intensive Care Med 5: 132-138, 2019.

6. Zhang A, Cai Y, Wang PF, Qu JN, Luo ZC, Chen XD, Huang B, Liu Y, Huang WQ, Wu J and Yin YH: Diagnosis and prognosis of neutrophil gelatinase-associated lipocalin for acute kidney injury with sepsis: A systematic review and meta-analysis. Crit Care 20 : 41-41, 2016.

7. Mishra J, Ma Q, Prada A, Mitsnefes M, Zahedi K, Yang J, Barasch $J$ and Devarajan P: Identification of neutrophil gelatinase-associated lipocalin as a novel early urinary biomarker for ischemic renal injury. J Am Soc Nephrol 14: 2534-2543, 2003.

8. Chakraborty S, Kaur S, Guha S and Batra SK: The multifaceted roles of neutrophil gelatinase associated lipocalin (NGAL) in inflammation and cancer. Biochim Biophys Acta 1826: 129-169, 2012.
9. Haase M, Bellomo R, Devarajan P, Schlattmann P and Haase-Fielitz A; NGAL Meta-analysis Investigator Group: Accuracy of neutrophil gelatinase-associated lipocalin (NGAL) in diagnosis and prognosis in acute kidney injury: A systematic review and meta-analysis. Am J Kidney Dis 54: 1012-1024, 2009.

10. Bone RC, Balk RA, Cerra FB, Dellinger RP, Fein AM, Knaus WA, Schein RM and Sibbald WJ: Definitions for sepsis and organ failure and guidelines for the use of innovative therapies in sepsis. The ACCP/SCCM consensus conference committee. American college of chest physicians/society of critical care medicine. Chest 101: 1644-1655, 1992.

11. Levy MM, Fink MP, Marshall JC, Abraham E, Angus D, Cook D, Cohen J, Opal SM, Vincent JL and Ramsay G; SCCM/ESICM/ACCP/ATS/SIS: 2001 SCCM/ESICM/ACCP/ATS/SIS international sepsis definitions conference. Crit Care Med 31: 1250-1256, 2003.

12. Dellinger RP, Levy MM, Rhodes A, Annane D, Gerlach H, Opal SM, Sevransky JE, Sprung CL, Douglas IS, Jaeschke R, et al: Surviving sepsis campaign: International guidelines for management of severe sepsis and septic shock, 2012. Intensive Care Med 39: 165-228, 2013

13. Khawaja S, Jafri L, Siddiqui I, Hashmi M and Ghani F: The utility of neutrophil gelatinase-associated Lipocalin (NGAL) as a marker of acute kidney injury (AKI) in critically ill patients. Biomark Res 7: 4, 2019.

14. Mehta RL, Kellum JA, Shah SV, Molitoris BA, Ronco C, Warnock DG and Levin A; Acute Kidney Injury Network: Acute kidney injury network: Report of an initiative to improve outcomes in acute kidney injury. Crit Care 11: R31, 2007.

15. Kellum JA and Lameire N; KDIGO AKI Guideline Work Group: Diagnosis, evaluation, and management of acute kidney injury: A KDIGO summary (Part 1). Crit Care 17: 204, 2013.

16. Whiting PF, Rutjes AW, Westwood ME, Mallett S, Deeks JJ, Reitsma JB, Leeflang MM, Sterne JA and Bossuyt PM; QUADAS-2 Group: QUADAS-2: A revised tool for the quality assessment of diagnostic accuracy studies. Ann Intern Med 155: 529-536, 2011.

17. Higgins JP and Green S (eds): Cochrane Handbook for Systematic Reviews of Interventions. Version 5.0.1, 2009.

18. Arends LR, Hamza TH, van Houwelingen JC, Heijenbrok-Kal MH, Hunink MG and Stijnen T: Bivariate random effects meta-analysis of ROC curves. Med Decis Making 28: 621-638, 2008.

19. Sterne JA, Sutton AJ, Ioannidis JP, Terrin N, Jones DR, Lau J, Carpenter J, Rücker G, Harbord RM, Schmid CH, et al: Recommendations for examining and interpreting funnel plot asymmetry in meta-analyses of randomised controlled trials. BMJ 343: d4002, 2011.

20. Yang YB, Li YH, Chen XQ, Lv LH and Mei WL: Diagnostic value of serum NGAL and CysC levels in sepsis patients with acute kidney injury. Zhejiang Med J 41: 1025-1029, 2019.

21. RQ L, ZY M, HG C and ZC L: Diagnostic efficacy of serum NGAL, KIM-1 and Cys-C for acute kidney injury in sepsis patients. China Med Herald 16: 128-131, 2019.

22. da Rocha EP, Yokota LG, Sampaio BM, Cardoso Eid KZ, Dias DB, de Freitas FM, Balbi AL and Ponce D: Urinary neutrophil gelatinase-associated lipocalin is excellent predictor of acute kidney injury in septic elderly patients. Aging Dis 9: 182-191, 2018.

23. Zheng J, He HL and Zhang GY: Neutrophil gelatinase-associated lipocalin as diagnosis biomarker for acute renal injury in pediatric patients with sepsis in intensive care unit. J Third Mil Med Univ 39: 196-200, 2017.

24. Shang Y, Li J, Zhang J, Wang W, Qiao Y and Ren X: Predictive performance of neutrophil gelatinase-associated lipocalin (NGAL) in acute kidney injury in septic patients. Chin J Emerg Med 26: 538-543, 2017.

25. Md Ralib A, Mat Nor MB and Pickering JW: Plasma neutrophil gelatinase-associated lipocalin diagnosed acute kidney injury in patients with systemic inflammatory disease and sepsis. Nephrology (Carlton) 22: 412-419, 2017.

26. Patel ML, Sachan R, Shyam R, Kumar S, Kamal R and Misra A: Diagnostic accuracy of urinary neutrophil gelatinase-associated lipocalin in patients with septic acute kidney injury. Int J Nephrol Renovasc Dis 9: 161-169, 2016.

27. Huang CY, Shih CC, Chung K, Kao KC and Wu HP: Predictive value of plasma neutrophil gelatinase-associated lipocalin for acute renal failure in patients with severe sepsis. J Chin Med Assoc 79: 428-434, 2016.

28. Zhang JG, Zhang DH, Zhu HP and Liu S: The clinical value of NGAL in the early diagnosis of sepsis-induced kidney injury. China Pract Med 10: 46-47, 2015. 
29. Niu KY, Yang F, Yang XY, Ye J and Cheng JJ: Diagnostic value of urinary liver-type fatty acid binding proteins and urinary neutrophil gelatinase-associated lipocalin in severe sepsis patients with acute kidney injury. Clin Focus 5: 536-539, 2015.

30. Nga HS, Medeiros P, Menezes P, Bridi R, Balbi A and Ponce D: Sepsis and AKI in clinical emergency room patients: The role of urinary NGAL. Biomed Res Int 2015: 413751, 2015.

31. Meng DL, Xing HB, Mao RS, et al: Predictive value of neutrophil gelatinase-associated lipocalin for acute kidney injury patients with sepsis. Zhongguo Jijiu Yixue: 224-229, 2015.

32. Hjortrup PB, Haase N, Treschow F, Moller MH and Perner A: Predictive value of NGAL for use of renal replacement therapy in patients with severe sepsis. Acta Anaesthesiol Scand 59: 25-34, 2015.

33. Zhou HQ, Chen MQ, Zhang HD and Wang X: sTREM-1 and NGAL levels for the early diagnosis of sepsis complicated by acute kidney injury. J Clin Exp Med 21: 1773-1775, 2014.

34. Wang XH and Zhang T: The value of NGAL in early diagnosis of acute kidney injury in children with sepsis. Pract Prev Med 21: 745-747, 2014.

35. Wang HX, Mu HB, Zheng RQ, Lin H, Yu JQ and Wu XY: Early diagnosis of neutrophil gelatinase-associated apolipoprotein in patients with acute kidney injury in sepsis. Shiyong Linchuang Yiyao Zazhi 18: 183-184, 2014

36. Fan H, Zhao Y, Zhu JH and Song FC: Urine neutrophil gelatinase-associated lipocalin in septic patients with and without acute kidney injury. Ren Fail 36: 1399-1403, 2014.

37. Xing HB, Lv T, Sheng PP, Chen JD, Mao RS and Li D: The diagnostic value of new biomarkers in sepsis patients with acute kidney injury. Zhongguo Jijiu Yixue 33: 507-510, 2013.

38. de Geus HR, Betjes MG, Schaick RV and Groeneveld JA: Plasma NGAL similarly predicts acute kidney injury in sepsis and nonsepsis. Biomark Med 7: 415-421, 2013.

39. Camou F, Oger S, Paroissin C, Guilhon E, Guisset O, Mourissoux G, Pouyes H, Lalanne T and Gabinski C: Plasma neutrophil gelatinase-associated lipocalin (NGAL) predicts acute kidney injury in septic shock at ICU admission. Ann Fr Anesth Reanim 32: 157-164, 2013 (In French).

40. Aydoğdu M, Gürsel G, Sancak B, Yeni S, Sarı G, Taşyürek S, Türk M, Yüksel S, Senes M and Ozis TN: The use of plasma and urine neutrophil gelatinase associated lipocalin (NGAL) and Cystatin $\mathrm{C}$ in early diagnosis of septic acute kidney injury in critically ill patients. Dis Markers 34: 237-246, 2013.

41. El-Farghali OG, El-Raggal NM, Mahmoud NH and Zaina GA: Serum neutrophil gelatinase-associated lipocalin as a predictor of acute kidney injury in critically-ill neonates. Pak J Biol Sci 15 231-237, 2012
42. Yan J, Xu HY, Zang D, Liang FM and Yang T: Significance of early diagnosis of urinary neutrophil gelatinase-associated lipocalin and urinary interleukin-18 in patients with sepsis complicated with acute kidney injury. Suzhou Univ J Med Sci 31: 785-788, 2011.

43. Shapiro NI, Trzeciak S, Hollander JE, Birkhahn R, Otero R, Osborn TM, Moretti E, Nguyen HB, Gunnerson K, Milzman D, et al: The diagnostic accuracy of plasma neutrophil gelatinase-associated lipocalin in the prediction of acute kidney injury in emergency department patients with suspected sepsis. Ann Emerg Med 56: 52-59.e1, 2010.

44. Mårtensson J, Bell M, Oldner A, Xu S, Venge P and Martling CR: Neutrophil gelatinase-associated lipocalin in adult septic patients with and without acute kidney injury. Intensive Care Med 36: 1333-1340, 2010.

45. Li PZ and Xu WX: Prediction of acute kidney injury complicated by sepsis with neutronphil gelatinase-associated lipocalin as an early marker. Chin J Lab Med 33: 492-496, 2010.

46. Shapiro DE: The interpretation of diagnostic tests. Stat Methods Med Res 8: 113-134, 1999.

47. Törnblom S, Nisulal S, Petäjä L, Vaara ST, Haapio M, Pesonen E and Pettilä V; FINNAKI study group: Urine NGAL as a biomarker for septic AKI: A critical appraisal of clinical utility-data from the observational FINNAKI study. Ann Intensive Care 10: 51 , 2020.

48. Seliger SL, Davis C and Stehman-Breen C: Gender and the progression of renal disease. Curr Opin Nephrol Hypertens 10: 219-225, 2001.

49. Mori K, Lee HT, Rapoport D, Drexler IR, Foster K, Yang J, Schmidt-Ott KM, Chen X, Li JY, Weiss S, Mishra J, et al: Endocytic delivery of lipocalin-siderophore-iron complex rescues the kidney from ischemia-reperfusion injury. J Clin Invest 115: 610-621, 2005

50. de Geus HR, Woo JG, Wang Y, Devarajan P, Betjes MG, le Noble JL and Bakker J: Urinary neutrophil gelatinase-associated lipocalin measured on admission to the intensive care unit accurately discriminates between sustained and transient acute kidney injury in adult critically ill patients. Nephron Extra 1: 9-23, 2011.

This work is licensed under a Creative Commons Attribution-NonCommercial-NoDerivatives 4.0 International (CC BY-NC-ND 4.0) License. 